\title{
Femtosecond compression dynamics and timing jitter suppression in a terahertz-driven electron bunch compressor
}

\author{
E. C. Snively, ${ }^{1, *}$ M. A. K. Othman, ${ }^{1, \dagger}$ M. Kozina, ${ }^{1}$ B. K. Ofori-Okai, ${ }^{1}$ S. P. Weathersby ${ }^{1}$

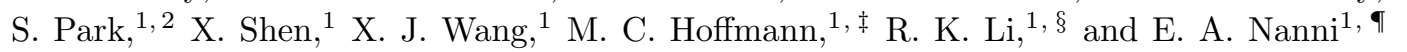 \\ ${ }^{1}$ SLAC National Accelerator Laboratory, 2575 Sand Hill Road, Menlo Park, California, 94025, USA \\ ${ }^{2}$ Stanford Institute for Materials and Energy Sciences, \\ SLAC National Accelerator Laboratory, Menlo Park, CA 94025, USA
}

\begin{abstract}
We present the first demonstration of THz-driven bunch compression and timing stabilization of a few-fC relativistic electron beam with kinetic energy of $2.5 \mathrm{MeV}$ using quasi-single-cycle strong field $\mathrm{THz}$ radiation in a shorted parallel-plate structure. Compression by nearly a factor of 3 produced a $39 \mathrm{fs}$ rms bunch length and a reduction in timing jitter by more than a factor of 2 , to $31 \mathrm{fs}$ rms, offering a significant improvement to beam performance for applications like ultrafast electron diffraction. This THz-driven technique provides a critical step towards unprecedented timing resolution in ultrafast sciences and other accelerator applications using femtosecond-scale electron beams.
\end{abstract}

PACS numbers:

Advances in electron-beam based ultrafast science continue to reach unprecedented sensitivity, with techniques like pump-probe ultrafast electron diffraction (UED) achieving sub-angstrom spatial resolution and temporal resolutions down to $100 \mathrm{fs}$ using electron bunch lengths of only tens of femtoseconds [1 3. The intense demand for ever shorter high-brightness electron beams has ignited a campaign to achieve fs-scale bunch lengths and timing stability through beam-wave interactions at $\mathrm{THz}$ frequencies 4 8, hand-in-hand with the advent of THz-based accelerator technology 9 12. THz-driven compression techniques in the relativistic regime, where space-charge effects are suppressed, could potentially enable production of high-brightness ultra-short bunches with sufficient charge to capture single shot images in UED, allowing characterization of irreversible processes at the time scale of atomic motion [13, 14. Bunch compression techniques using conventional radio frequency (RF) structures have succeeded in producing ultralow emittance few-fs beams [15] and represent a significant step towards achieving the high-brightness beams needed for single-shot UED. However, the interaction suffers from RF phase jitter, on the order of tens to hundreds of femtoseconds depending on the source stability [16 18], which can exacerbate the time-of-arrival jitter between compressed electron beam and reference pump laser.

THz-driven beam manipulation has been recognized as a promising candidate in the pursuit of few-fs beams with sub-fs timing resolution, because the inherent timing synchronization of all-optical control enables both bunch compression and reduction of beam timing jitter. Already, THz-driven beam manipulation has demonstrated dramatic compression in the sub-relativistic regime ( $<100 \mathrm{keV}$ electrons), reaching bunch lengths of tens of femtoseconds while reducing the timing jitter to a few femtoseconds [4, 6]. Beyond the synchronization benefit that comes with a laser-driven interaction, structures operating in the $\mathrm{THz}$ regime offer a host of advantages. For applications like bunch compression or transverse deflection, the higher frequency provides a more efficient time-dependent momentum kick compared to conventional RF manipulation. Using sub-wavelength structures for localized field enhancement [19, THz-driven streaking diagnostics have already demonstrated femtosecond, down to sub-femtosecond metrology of relativistic beams 20, 21. Additionally, the sub-mm length scale of the $\mathrm{THz}$ regime enables the use of small-footprint structures supporting strong synchronism for efficient beam manipulation while occupying only $\mathrm{mm}$-scale space on the beamline.

We present the results of a $\mathrm{THz}$-driven compression experiment performed at the SLAC MeV-UED facility at SLAC National Accelerator Laboratory using a shorted parallel-plate waveguide (PPWG) design [22]. Both the manipulation and characterization of the relativistic electron beam were accomplished through interaction with quasi-single-cycle $\mathrm{THz}$ pulses generated via optical rectification of $800 \mathrm{~nm}$ laser pulses 23. Using the shorted PPWG to couple the relativistic electron beam and an orthogonally propagating $<3 \mu \mathrm{J} \mathrm{THz}$ pulse, we produced a beam energy chirp resulting in compression by a factor of 2.7, with a minimum bunch length of $39 \pm 7 \mathrm{fs}$ rms. An equally important consequence of this interaction is the simultaneous improvement to the beam's shot-to-shot time-of-arrival (rms) stability by a factor of $>2.5$, in this case reducing the timing jitter to $31 \mathrm{fs}$ rms. This THz-driven compression experiment builds on the success of the THz-based streaking diagnostic developed at SLAC MeV-UED, which previously demonstrated sub-fs rms timing accuracy of few $\mathrm{MeV}$ beams 21. The compo- 
nents of the THz-driven bunch compression and timing stabilization setup are integrated with the THz-driven beam diagnostic, as shown in Fig. 1. The enhanced capabilities of these combined technologies are uniquely positioned to be directly applied to UED experiments.

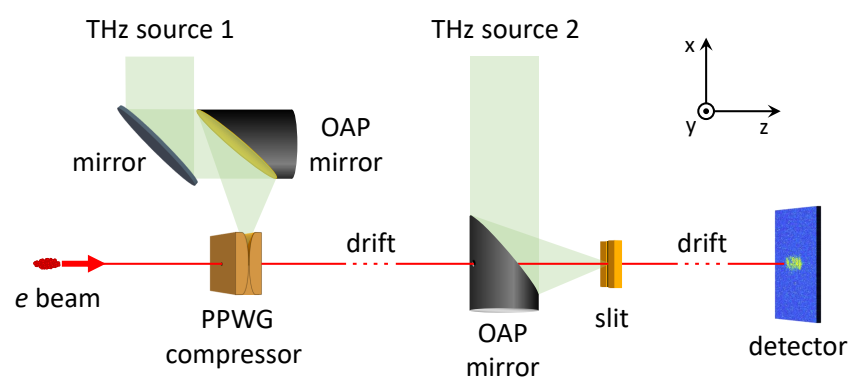

FIG. 1: Schematic of the in-vacuum components for the THzdriven compression and streaking setup. As the electron beam passes through the parallel plate waveguide (PPWG) compressor, the orthogonally propagating $\mathrm{THz}$ pulse imparts an energy chirp, resulting in velocity bunching during the subsequent drift. The beam then passes through an OAP mirror into collinear propagation with a second $\mathrm{THz}$ pulse as it is focused into a metallic slit. Here, the $\mathrm{THz}$ pulse imparts a transverse momentum kick to the beam which streaks the longitudinal profile onto the y-axis on a downstream imaging detector. $\mathrm{THz}$ source 1 is polarized along the z-axis; $\mathrm{THz}$ source 2 is polarized along the y-axis.

The Ti:Sapphire laser system at SLAC MeV-UED provided a $13 \pm 1 \mathrm{~mJ}$, near-IR $(800 \mathrm{~nm})$ pulse at $180 \mathrm{~Hz}$ which was split to drive the UV source, for electron beam generation in the S-band photocathode gun, and two separate $\mathrm{THz}$ sources, in which the pulse-front-tilt method was used for optical rectification in $\mathrm{LiNbO}_{3}$ crystals [24, 25]. A 70-30 beam splitter directed the primary IR pulse to $\mathrm{THz}$ source 1, dedicated to driving bunch compression, with the secondary pulse continuing on to $\mathrm{THz}$ source 2 for the streaking setup. Two translation stages controlled the timing of each $\mathrm{THz}$ source via adjustment of the IR path length. To produce the horizontally polarized $\mathrm{THz}$ field required for interaction in the compressor, the components of the corresponding pulse-front-tilted optical rectification setup were mounted to a vertical breadboard. The resulting quasi-single-cycle $\mathrm{THz}$ pulse was transported into a vacuum chamber on the beamline using a pair of off-axis parabolic mirrors (OAP) to first collimate and then focus the pulse into the horn of the compressor structure, as shown in Fig. 11. Downstream, the second $\mathrm{THz}$ source produced a quasi-single-cycle pulse polarized along the y-axis, which was similarly collimated and transported into a second vacuum chamber using a pair of OAPs. In this case, the final OAP was mounted on the beamline axis such that a small $2.5 \mathrm{~mm}$ hole in the mirror allowed the electron beam to pass through into co-propagation with the $\mathrm{THz}$ pulse as it was focused into a metallic PPWG "slit" structure for streaking the beam [21].

The $2.856 \mathrm{GHz}$ photocathode gun on the SLAC MeVUED beamline supplied a relativistic electron beam with 2.5 MeV kinetic energy and sub-10 fC total charge. After initial collimation with a focusing solenoid, the beam was aligned through a pinhole and then focused with a second solenoid to a $\sim 40 \mu \mathrm{m}$ rms spot size in the compressing structure. The bunch length and timing jitter of the uncompressed beam were $105 \pm 19$ fs rms and 76 fs rms, respectively, measured by the THz-driven streaking diagnostic $1 \mathrm{~m}$ downstream of the compressor.

The THz-driven compression technique presented here utilized a PPWG structure that benefits from two key design enhancements. Where the $\mathrm{THz}$ pulse entered the structure, an exponentially-tapered adiabatic horn was matched to the free-space $\mathrm{THz}$ beam profile, significantly improving the coupling efficiency and allowing dispersion-free focusing of the $\mathrm{THz}$ pulse into the PPWG at the center of the structure. Using the coordinate system of the compressor diagram in Fig. 2, the adiabatic horn focused the z-component of the quasi-single-cycle $\mathrm{THz}$ pulse as it propagated along the x-axis, overcoming the free space diffraction limit and resulting in significant field enhancement between the parallel plates aligned in the $\mathrm{x}-\mathrm{y}$ plane. Within the $210 \mu \mathrm{m}$ gap of the PPWG structure, the addition of a copper short, located at the

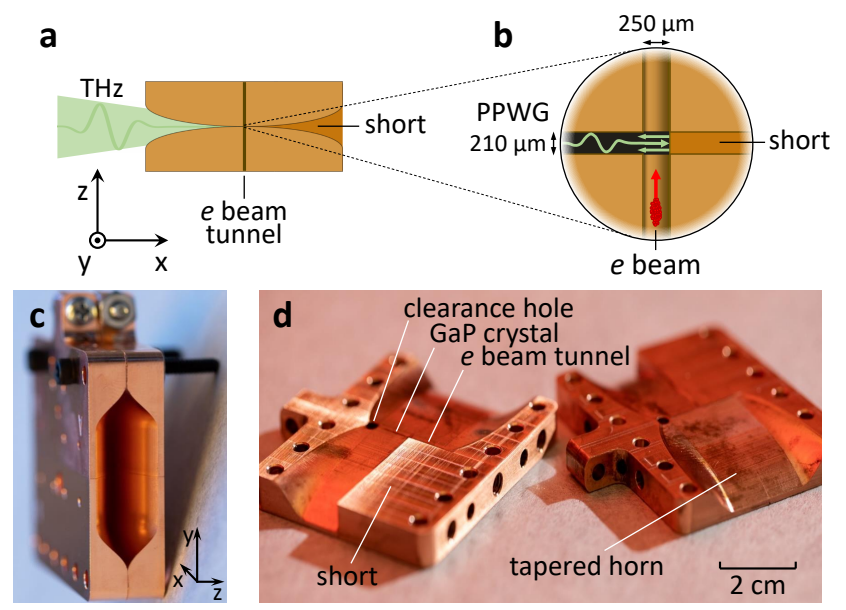

FIG. 2: (a) Diagram of the compressor structure cross-section, showing the exponentially tapered horn with incoming $\mathrm{THz}$ pulse polarized along the z-axis. (b) A close-up view of the interaction region, showing the $250 \mu \mathrm{m}$ diameter beam tunnel and the PPWG with $210 \mu \mathrm{m}$ gap. A short reflects the incoming $\mathrm{THz}$ pulse as the beam passes through. (c) A photograph of the assembled structure showing the adiabatic horn opening. (d) A photograph of the disassembled structure before the final etch. The structure is translated along the y-axis to switch between compressor "on" with beam passing through the beam tunnel and compressor "off" with beam passing through the $2.54 \mathrm{~mm}$ diameter clearance hole. A GaP crystal, mounted between the beam tunnel and clearance hole, enabled in-situ electro-optic sampling of the $\mathrm{THz}$ field. 
edge of the beam tunnel (125 $\mu \mathrm{m}$ from beam axis), produced a superposition of forward and reflected $\mathrm{THz}$ field in the vicinity of the passing electron beam, increasing the parallel electric field driving the energy chirp, while reducing the magnetic field which imparted an undesirable transverse momentum kick.

As the electron beam traversed the PPWG gap, the integrated Lorentz force experienced by particles at different positions along the bunch length was determined by the temporal overlap with the $\mathrm{THz}$ field. Ideally, the beam was injected at a phase with maximum $\mathrm{THz}$ electric field gradient, typically near a zero-crossing in the waveform, which produced the strongest near-linear longitudinal energy chirp, resulting in efficient velocity bunching of the beam. The temporal profile of the $\mathrm{THz}$ pulse determined the phase acceptance window over which the beam received an energy chirp with the correct sign for bunch compression. For beams arriving within this window, the $\mathrm{THz}$ electric field gradient increased the energy of particles at the tail of the beam relative to particles at the head of the beam, as shown in Fig. 3p. It follows directly that within this window, the energy of a "late" bunch was increased relative to an "early" bunch, such that the compressor interaction also reduced the shotto-shot beam timing jitter after a subsequent drift. This effect takes advantage of the inherent timing synchronization between the $\mathrm{THz}$ pulse and electron beam generated using the same initial laser pulse.

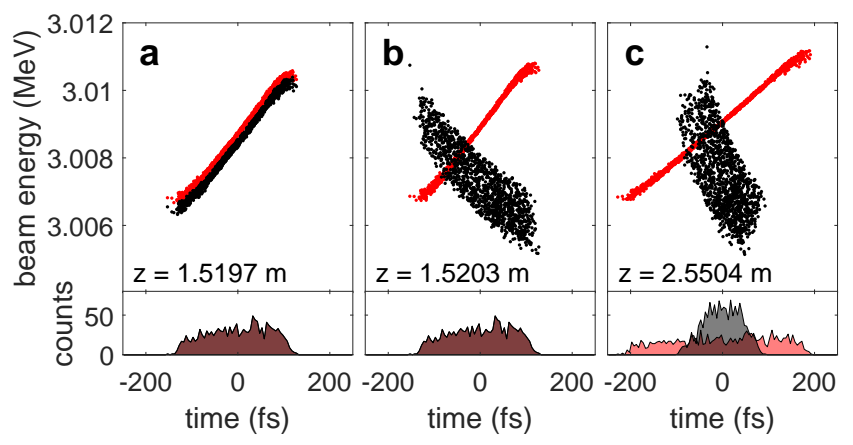

FIG. 3: Simulated longitudinal phase space of the beam at different points along the beamline, with compressor "off" shown in red and compressor "on" in black. (a) Just before entering the compressor, the two cases show the same distribution. (b) As it nears the exit of the compressor, the beam that interacted with the $\mathrm{THz}$ pulse (black) has accumulated an energy chirp of opposite sign. During the drift, higher energy electrons move forward relative to the center of the bunch, while lower energy electrons move back. (c) As a result, downstream at the streaking slit, the uncompressed beam (red) has increased in length, while the compressed beam (black) has become shorter through velocity bunching. The projected bunch length is shown at the bottom of each plot.

In addition to producing the energy chirp necessary for velocity bunching, the Lorentz force within the PPWG interaction region produced a time-dependent transverse deflection of the beam. The shorted PPWG configuration was chosen to reduce this transverse deflection and defocusing caused by the magnetic field, with the superposition of the forward and reflected $\mathrm{THz}$ wave producing constructive interference of the transverse electric field while the magnetic field was partially canceled 22. At the local maxima in deflection, the shot-to-shot beam pointing jitter increased to $30 \mu \mathrm{rad}$ rms from the 10 $\mu \mathrm{rad} \mathrm{rms}$ jitter without $\mathrm{THz}$ interaction. The interaction phase for maximum compression, near a local maximum in deflection, produced a shot-to-shot pointing jitter of up to $50 \mu \mathrm{rad} \mathrm{rms}$. Steering magnets placed after the compression chamber were used to compensate for the compressor-induced deflection, ensuring consistent alignment through the streaking diagnostic.

We observed both compression of the electron bunch length and a reduction in the shot-to-shot timing jitter using the THz-driven streaking setup positioned 1 $m$ downstream from the compression interaction. Here the longitudinal profile of the electron beam was projected onto the vertical axis through the time-dependent transverse momentum kick imparted to the beam by a co-propagating $\mathrm{THz}$ pulse in a metallic slit [21. The direction and magnitude of the deflection was determined by the temporal profile of the $\mathrm{THz}$ pulse. A calibration of femtoseconds per pixel was directly obtained by scanning the streaking $\mathrm{THz}$ pulse arrival time and mapping the corresponding deflection of the beam centroid on the phosphor screen placed $2 \mathrm{~m}$ downstream of the streaking interaction.

To extract the beam characteristics from a single shot image of the charge profile after streaking, we compare the measured distribution to the distribution produced by assuming a Gaussian longitudinal profile for a test beam which is then mapped into a "projected distribution" using the known streaking calibration curve. The length and arrival time of the Gaussian test beam are allowed to vary in order to find the best fit of the simulated distribution to the actual projected beam image. This method effectively deals with the irregular charge distributions produced when the initial longitudinal beam profile fills the region mapped out by the THz-driven transverse deflection, causing the charge to "pile-up" at the ends of the projected distribution.

In Figure 4 a-d, we show examples of single shot beam images alongside their corresponding projected distribution, with the background subtracted, and the fit produced through the test beam method. These examples highlight two cases, in which the compression interaction is first turned off and then turned on to produce a maximally compressed bunch. In Fig. 4p-l, we plot the bunch length and time-of-arrival for 1000 shots of the uncompressed beam, indicating a mean bunch length of $105 \pm 19$ fs rms and timing jitter of $76 \mathrm{fs}$ rms, and for 1000 shots of the compressed beam, indicating a mean bunch length of $39 \pm 7 \mathrm{fs}$ rms and timing jitter of $31 \mathrm{fs}$ rms. The reduc- 

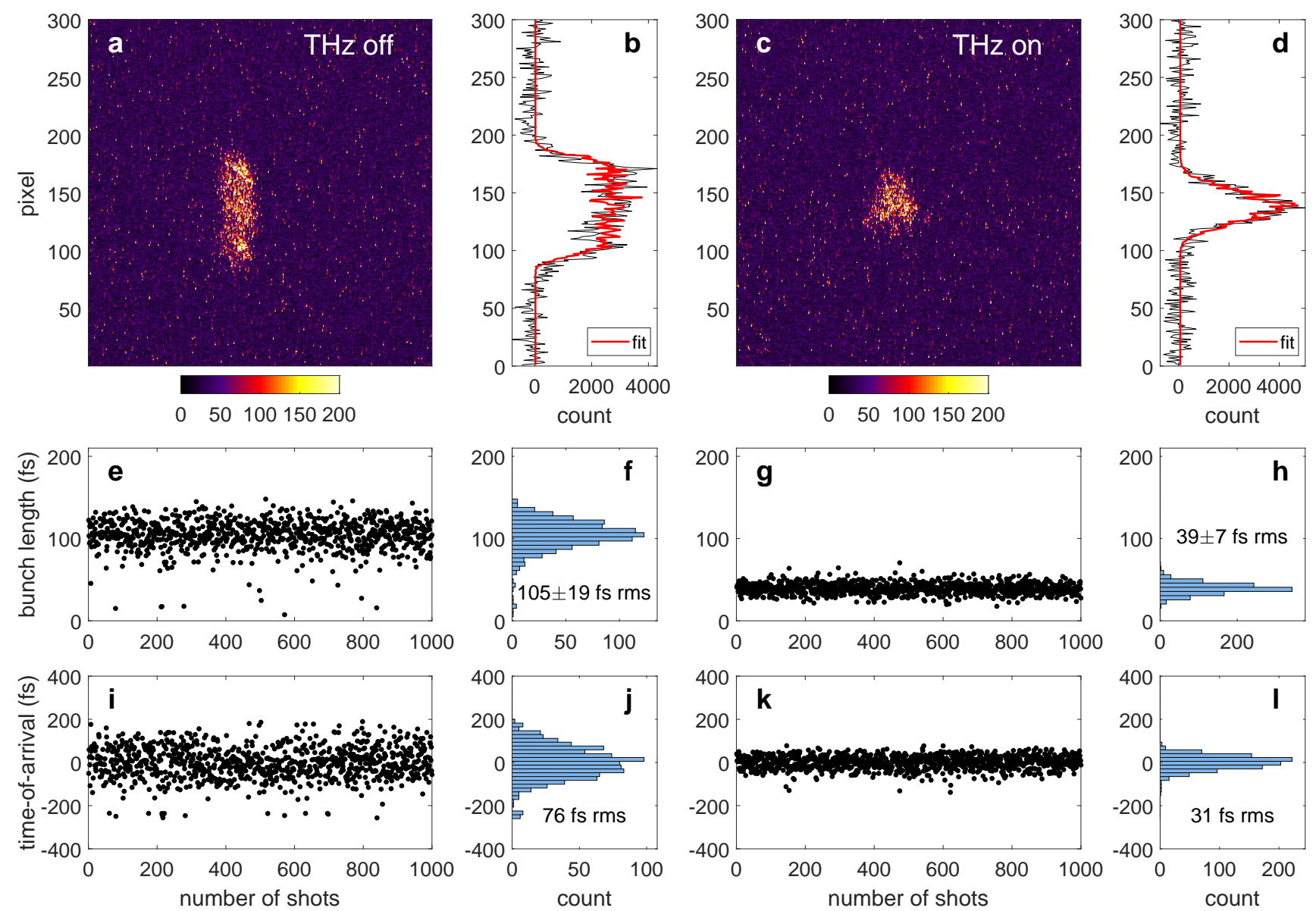

FIG. 4: Comparison of the uncompressed beam to the maximally compressed beam. Single shot beam images in (a) and (c) show examples with THz-driven compression "off" and "on", respectively, with the corresponding fit to their projected distribution shown in (b) and (d). (e)-(f) show the bunch length of the uncompressed beam over 1000 shots, with an average bunch length of $105 \pm 19 \mathrm{fs}$ rms. (g)-(h) show the bunch length of the compressed beam over 1000 shots, indicating an average bunch length of $39 \pm 7$ fs rms. (i)-(j) show the time-of-arrival of the uncompressed beam over 1000 shots, with a timing jitter of $76 \mathrm{fs}$ rms. (k)-(l) show the time-of-arrival of the compressed beam over 1000 shots, giving a reduced timing jitter of 31 fs rms.

tion in timing jitter by a factor of 2.5 is slightly smaller than the measured compression factor of 2.7. However, similar measurements of 1000 shots taken at neighboring interaction phases near the minimum bunch length show timing jitters as low as $24 \mathrm{fs}$ rms, as shown in Fig. 5a, indicating jitter reduction by up to a factor of 3.2.

In Fig. 5 a, we plot the results of a scan of $\mathrm{THz}$ arrival time within the compressing structure, including the corresponding bunch length, timing jitter and transverse deflection. These parameters vary in time according to the quasi-single-cycle profile of the driving $\mathrm{THz}$ field. The deflection data shown in Fig. 5 a was collected in a separate scan, independent of the streaking setup, with finer temporal resolution. The width of the line reflects the standard deviation in beam centroid position along the $\mathrm{x}$-axis in the lab frame. Because the compressor-induced transverse deflection of the beam necessitated realignment between each streaking measurement, the full set of bunch length and timing jitter measurements was acquired over a couple hours. During this time, a slow drift in the relative time-of-arrival resulted in a cumulative scaling uncertainty on the order of 1 ps per hour.

The profile of the $\mathrm{THz}$ pulse measured via $\mathrm{EO}$ sampling in the un-shorted section of the PPWG is shown in Fig. 5b. These EO measurements were used to generate a simulated profiled of the $\mathrm{THz}$ field in the shorted PPWG section with Ansoft HFSS software 22. Simulations of the electron beam dynamics were performed with the GPT simulation package [26], beginning in the photocathode gun and including the compressor field maps imported from HFSS. Given our measured beam energy and uncompressed bunch length of $105 \mathrm{fs}$ rms, GPT simulation shows that the peak field sampled by the beam within the compressor must be around $100 \mathrm{MV} / \mathrm{m}$ in order to reproduce the compressed bunch length of $39 \mathrm{fs}$ rms as measured at the streaking slit. This value is con- 

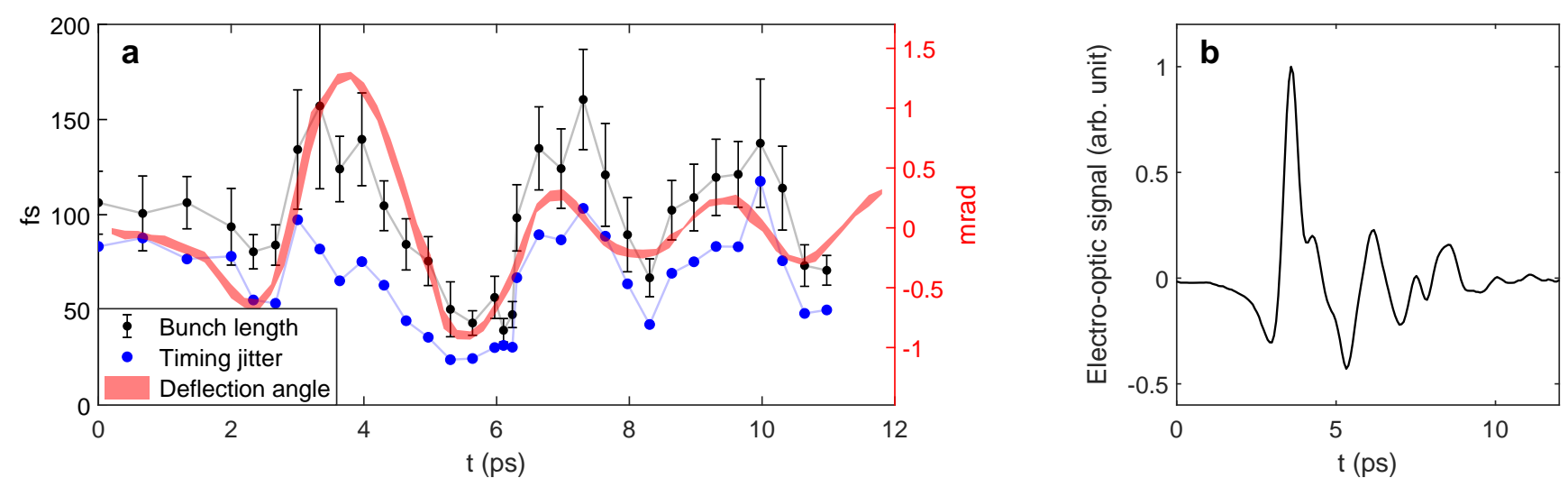

FIG. 5: (a) Scan of the bunch length and timing jitter of the electron beam, along with the deflection angle in the x-z plane (scale shown on right axis), as a function of the THz pulse arrival time within the structure. The quasi-single-cycle profile of the driving $\mathrm{THz}$ field is evident in the time-dependent oscillations of the beam characteristics, each showing a parallel change in sign and magnitude. (b) Temporal profile of the THz pulse measured within the PPWG structure via electro-optic sampling with the GaP crystal. This measurement characterizes the $\mathrm{THz}$ field in the un-shorted region of the PPWG structure. The ringing apparent after $\sim 6 \mathrm{ps}$ is an aftifact from the EO sampling crystal, not the true waveform.

sistent with our expected field enhancement, given the THz pulse energy and structure design $[22$. These simulated values correspond to an incoming beam energy chirp of $1.7 \mathrm{keV}$ per $100 \mathrm{fs}$, and exiting chirp of $-1.3 \mathrm{keV}$ per $100 \mathrm{fs}$, and indicate that maximum compression of the $2.5 \mathrm{MeV}$ bunch occurred approximately $70 \mathrm{~cm}$ downstream of the streaking slit, where the beam reached a minimum bunch length of $\sim 32 \mathrm{fs}$ rms. The actual bunch compression achieved in our experiment was primarily limited by the available $\mathrm{THz}$ energy, with the measurement of that compression further limited by the location of our streaking diagnostic. Eventually, the compression achieved through this shorted PPWG technique would also be limited by the increase to the slice energy spread of the beam.

The transverse deflection evident in our measurements and in GPT simulations of the compressor interaction is predominantly along the $\mathrm{x}$-axis. The measured deflection along the $\mathrm{y}$-axis mirrored the $\mathrm{x}$-deflection but with smaller magnitude, reaching only $250 \mu \mathrm{rad}$ where the $\mathrm{x}$ deflection peaked to $1.27 \mathrm{mrad}$. The magnitude of the measured y-deflection is consistent with simulations, but the GPT model predicts $\mathrm{x}$-deflection that is a factor of two larger than observed in measurements. Differences in the position of the beam within the tunnel cross-section are a likely source of discrepancy between our measurements and simulation, causing the beam to sample a different region of the shorted field profile compared to the on-axis trajectory assumed in simulation. This inhomogeneity caused by the short in the PPWG structure is key to the reduction in the transverse deflection, and required careful optimization of the distance between the short and beam axis for the phase of the forward and reflected magnetic field to add destructively along the beam path 22].

A more straight-forward design for mitigating the transverse momentum kick is a dual feed structure that utilizes two counterpropagating $\mathrm{THz}$ pulses. This technique has been successfully demonstrated for THzdriven acceleration, compression and streaking of a subrelativistic beam [6]. While this method offers advantages in the flexibility and fidelity of the $\mathrm{THz}$ field superposition, it introduces the additional challenge of requiring either a second $\mathrm{THz}$ source, or $\mathrm{THz}$ splitting optics with additional beam transport. In the development of next-generation single-feed PPWG structures, a split waveguide design, in which the beam tunnel passes through two PPWG interaction regions, could provide added flexibility for optimization of the energy chirp while canceling the induced deflection. This alternate design could also improve the uniformity of the field in the vicinity of the beam, reducing the slice energy spread growth predicted for the current design.

In summary, we have shown a compression interaction, driven by a laser-generated $\mathrm{THz}$ pulse in a parallel plate waveguide, that reduces the bunch length while acting to correct the timing jitter of the relativistic beam, stabilizing its time-of-arrival with respect to a reference laser pulse. The simultaneous compression to a $39 \mathrm{fs} \mathrm{rms}$ bunch length and $31 \mathrm{fs}$ rms timing jitter provides a significant benefit to performance for electron-beam based ultrafast science, given that the overall temporal resolution of a measurement is dependent on the bunch length and timing jitter added in quadrature. The transverse deflection of the beam induced by this compression interaction is easily corrected using steering magnets and introduces only a minimal increase to the beam pointing jitter. The magnitude of compression achieved through this tech- 
nique could be directly improved by increasing the input $\mathrm{THz}$ pulse energy, with state-of-the-art $\mathrm{THz}$ sources already providing greater pulses energies by more than an order of magnitude [27, 28. This demonstration of $\mathrm{THz}-$ driven compression and timing stabilization is a critical step towards achieving the ultra-short electron beams that could enable UED measurements at the attosecond scale. More broadly, electron beams conditioned through this technique are advantageous for a range of accelerator applications, like pump-probe ultrafast electron scattering and external injection in laser-driven accelerators that require few-fs bunch lengths and timing stability 2931 .

The authors acknowledge M. Cardoso, A. Sy, and A Haase for structure fabrication. This research has been supported by the U.S. Department of Energy (DOE) under Contract No. DE-AC02-76SF00515. The SLAC $\mathrm{MeV}-\mathrm{UED}$ program is supported in part by DOE Basic Energy Sciences (BES) Scientific User Facilities Division and SLAC UED/UEM program development: DEAC02-05CH11231. M. K. and M. C. H. are supported by the DOE Office of Science, BES, award no. 2015SLAC100238-Funding.

E. C. S., M. A. K. O., and M. K. contributed equally to this work.

* Electronic address: esnively@slac.stanford.edu

† Electronic address: mothman@slac.stanford.edu

¥ Electronic address: hoffmann@slac.stanford.edu

$\S$ Electronic address: lirk@tsinghua.edu.cn

ฯ Electronic address: nanni@slac.stanford.edu

[1] S. P. Weathersby, G. Brown, M. Centurion, T. F. Chase, R. Coffee, J. Corbett, J. P. Eichner, J. C. Frisch, A. R. Fry, M. Gühr, and N. Hartmann. Review of Scientific Instruments, 86.7, (2015).

[2] E. M. Mannebach, R. Li, K. A. Duerloo, C. Nyby, P. Zalden, T. Vecchione, F. Ernst, A. H. Reid, T. Chase, X. Shen, and S. Weathersby. Nano letters, 15(10), 6889$6895,(2015)$.

[3] J. Yang, M. Guehr, T. Vecchione, M. S. Robinson, R. Li, N. Hartmann, X. Shen, R. Coffee, J. Corbett, A. Fry, and K. Gaffney. Nature communications 7, 11232, (2016).

[4] C. Kealhofer, W. Schneider, D. Ehberger, A. Ryabov, F. Krausz, and P. Baum. Science 352(6284), 429-433 (2016).

[5] E. Curry, S. Fabbri, J. Maxson, P. Musumeci, and A. Gover, Phys. Rev. Lett. 120(9), 094801 (2018).

[6] D. Zhang, A. Fallahi, M. Hemmer, X. Wu, M. Fakhari, Y. Hua, H. Cankaya, A. L. Calendron, L. E. Zapata, N. H. Matlis, and F. X. Kärtner, Nat. Photonics 12(6), 336 (2018).

[7] L. Zhao, T. Jiang, C. Lu, R. Wang, Z. Wang, P. Zhu, Y. Shi, et al. Phys. Rev. Accel. Beams 21(8), 082801 (2018).

[8] D. Ehberger, A. Ryabov, and P. Baum. Physical review letters 121(9), 094801 (2018).

[9] L. J. Wong, A. Fallahi, and F. X. Kärtner. Optics express 21(8), 9792-9806, (2013).
[10] L. Wimmer, G. Herink, D. R. Solli, S. V. Yalunin, K. E. Echternkamp, and C. Ropers. Nature Physics 10(6), 432, (2014).

[11] E. A. Nanni, W. R. Huang, K. H. Hong, K. Ravi, A. Fallahi, G. Moriena, R. D. Miller, and F. X. Kärtner. Nature communications 6, 8486, (2015).

[12] W. R. Huang, A. Fallahi, X. Wu, H. Cankaya, A. L. Calendron, K. Ravi, D. Zhang, E. A. Nanni, K. H. Hong, and F. X. Kärtner. Optica 3(11), 1209-1212, (2016).

[13] G. Sciaini, and R. D. Miller. Rep. Prog. Phys. 74(9), 096101 (2011).

[14] E. Hall, S. Stemmer, H. Zheng, Y. Zhu, and G. Maracas. US Department of Energy, Washington, DC (United States), 2014.

[15] J. Maxson, D. Cesar, G. Calmasini, A. Ody, P. Musumeci, and D. Alesini. Phys. Rev. Lett. 118(15), 154802 (2017).

[16] P. Craievich, S. Di Mitri, M. Milloch, G. Penco, and F. Rossi. Physical Review Special Topics-Accelerators and Beams, 16, no. 9 (2013): 090401.

[17] R. Pompili, M. P. Anania, M. Bellaveglia, A. Biagioni, G. Castorina, E. Chiadroni, A. Cianchi, M. Croia, D. Di Giovenale, M. Ferrario, and F. Filippi. New Journal of Physics, 18, no. 8 (2016): 083033.

[18] R. K. Li and C. X. Tang. Nuclear Instruments and Methods in Physics Research Section A: Accelerators, Spectrometers, Detectors and Associated Equipment, 605, no. 3 (2009): 243-248.

[19] J. Fabiańska, G. Kassier and T. Feurer. Scientific Reports 4: 5645 (2014)

[20] L. Zhao, Z. Wang, C. Lu, R. Wang, C. Hu, P. Wang, J. Qi, et al. Phys. Rev. X 8(2), 021061 (2018).

[21] R. K. Li, M. C. Hoffmann, E. A. Nanni, S. H. Glenzer, M. E. Kozina, A. M. Lindenberg, et al. Phys. Rev. Accel. Beams 22(1), 012803 (2019).

[22] M. A. Othman, M. C. Hoffmann, M. K. Kozina, X. J. Wang, R. K. Li, and E. A. Nanni. arXiv preprint, arXiv:1905.02831 (2019).

[23] B. K. Ofori-Okai, M. C. Hoffmann, A. H. Reid, S. Edstrom, R. K. Jobe, R. K. Li, E. M. Mannebach, et al. J. Instrum. 13(06), P06014 (2018).

[24] J. Hebling, G. Almasi, I. Z. Kozma, and J. Kuhl. Opt. Express 10(21), 1161-1166 (2002).

[25] J. A. Fülöp, L. Pálfalvi, M. C. Hoffmann, and J. Hebling. Opt. express 19(16), 15090-15097 (2011).

[26] M. J. De Loos, and S. B. Van Der Geer. General Particle Tracer: A new 3D code for accelerator and beamline design. In 5th European Particle Accelerator Conference, (1996).

[27] J. A. Fülöp, Z. Ollmann, C. Lombosi, C. Skrobol, S. Klingebiel, L. Pálfalvi, F. Krausz, S. Karsch, and J. Hebling. Opt. Express 22(17), 20155-20163 (2014).

[28] C. Vicario, B. Monoszlai, and C. P. Hauri. Physical Review Letters, 112, no. 21 (2014): 213901.

[29] V. Malka, J. Faure, Y. A. Gauduel, E. Lefebvre, A. Rousse, and K. T. Phuoc. Nature physics, 4(6), 447 (2008).

[30] E. Esarey, C. B. Schroeder, and W. P. Leemans. Reviews of modern physics, 81, no. 3 (2009): 1229.

[31] R. J. England, R. J. Noble, K. Bane, D. H. Dowell, C. K. $\mathrm{Ng}$, J. E. Spencer, S. Tantawi, et al. Reviews of Modern Physics 86, no. 4 (2014): 1337. 